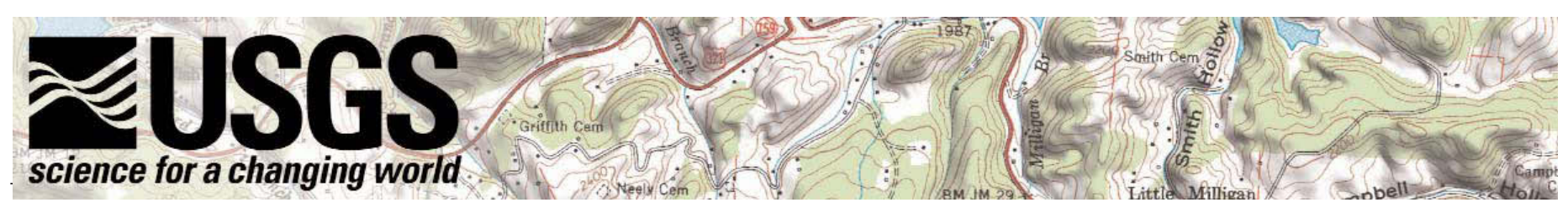

\title{
The National Map - Hydrography
}

\section{The Nation Needs The National Map}

Governments depend on a common set of base geographic information as a tool for economic and community development, land and natural resource management, and health and safety services. Emergency management and homeland security applications rely on this information. Private industry, nongovernmental organizations, and individual citizens use the same geographic data. Geographic information underpins an increasingly large part of the Nation's economy.

Available geographic data often:

Do not align with each other because layers are frequently created or revised separately,

Do not match across administrative boundaries because each producing organization uses different methods and standards, and

Are not up to date because of the complexity and cost of revision.

The U.S. Geological Survey (USGS) is developing The National Map as a seamless, continuously maintained, and nationally consistent set of online, public domain, geographic base information to address these issues. The National Map will serve as a foundation for integrating, sharing, and using other data easily and consistently.

In collaboration with other Federal, State, county, and local government agencies, the private sector, academia, and the public, the USGS will coordinate, integrate, and, where needed, produce and maintain base geographic data.

The National Map will include digital orthorectified imagery, elevation data, vector data for hydrography, transportation, boundary, and structure features, geographic names, and land cover information. The data will be the source of revised paper topographic maps.

\section{The National Hydrography Dataset}

The National Hydrography Dataset (NHD) is the surface water feature data component of The National Map. The initial implementation of the NHD was produced by combining the geometry of water features from USGS digital line graph data with river reach codes and other information developed by the Environmental Protection Agency (EPA). The USGS and the EPA are committed to a long-term partnership to improve and maintain the NHD. It includes information about naturally occurring and constructed bodies of water, paths through which water flows, and related entities. Attributes are attached to the feature information to convey classification (for example, perennial and intermittent), geographic name, position, length, area, and water flow direction. A reach code assigned to each feature uniquely identifies that feature and is a link through which other information can be related to the NHD. In addition to this geographic information, the dataset contains metadata and information that supports future updates and improvements to the data.

NHD data support numerous hydrographic applications, such as the following:

\section{Making maps. Positional and} descriptive data in the NHD enable users to plot and symbolize features.

C Geocoding observations. The NHD reach codes serve as "stream addresses" to link other attribute information and related features to NHD features.

C Modeling. Information about the direction of waterflow, when combined with other data, enables users to model

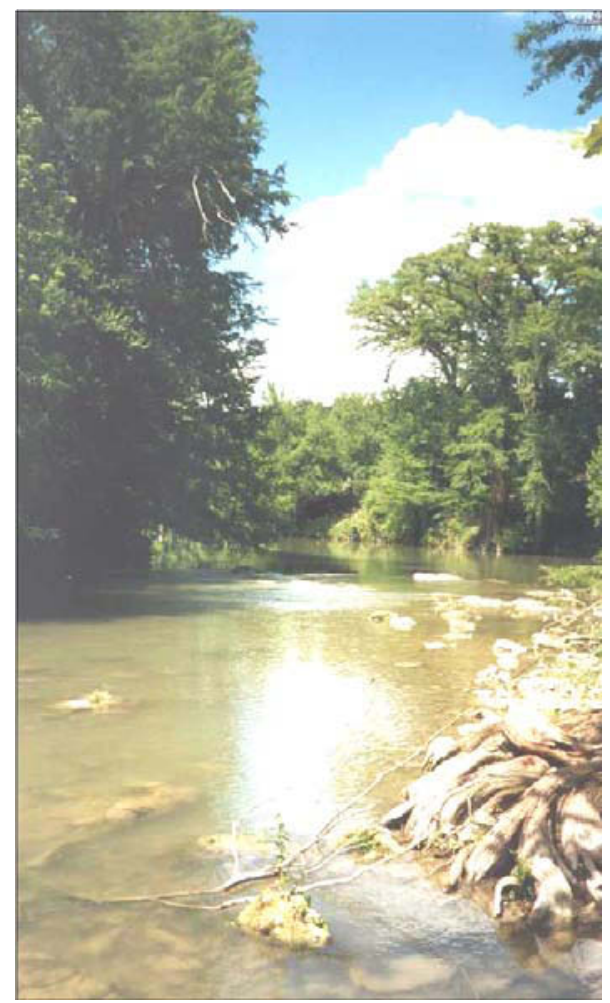

the transport of materials, such as contaminants, in hydrographic networks.

C Data maintenance. The visions of The National Map include partnerships to share the responsibilities for improving and updating geographic data such as the NHD. Unique identifiers and other information encoded in the NHD help to overcome technical problems of shared data maintenance.

\section{Data Availability and Plans}

The initial implementation of the NHD was based on the delineations of water features from 1:100,000-scale USGS topographic maps and the EPA's Reach File Version 3. Thus the content is cartographic in that features may be generalized in shape and complexity, displaced to accommodate other content, combined, and/or dropped 


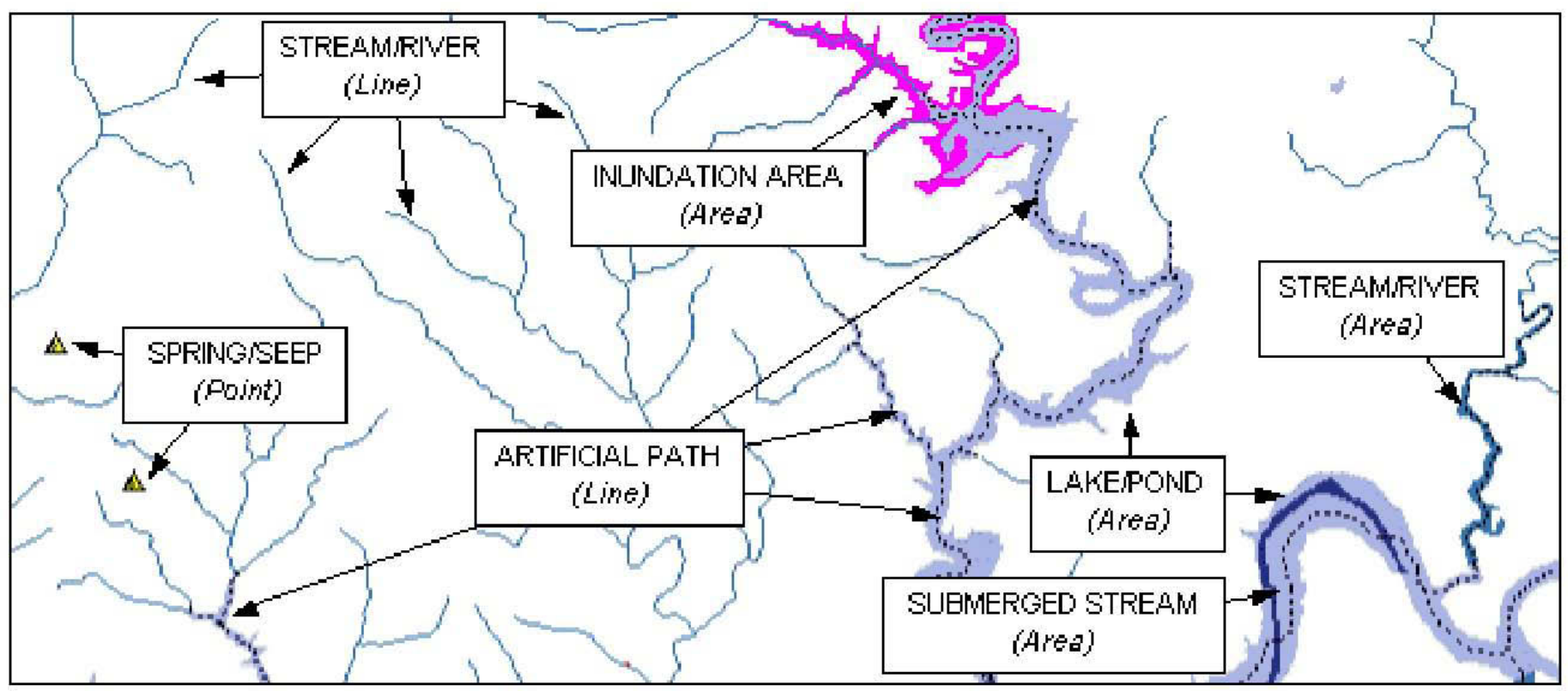

National Hydrography Dataset features include points, lines, and areas that represent standing and flowing surface water. Artificial paths are implied connections that provide continuity for modeling waterflow.

owing to the space constraints of the medium-scale maps.

High-resolution data, typically developed from 1:24,000-scale USGS topographic maps (1:63,360-scale in Alaska), 1:12,000scale digital orthophotos, or larger scale sources provided by local cooperators, are being developed according to the availability of matching resources from NHD partners.

Among Federal agencies, Department of the Interior Bureaus support the development of high-resolution data through the High-Priority Digital Base Data Program managed by the USGS. Much of this work has been dedicated to coverage of Alaska. The U.S. Forest Service (USFS) is working with the USGS to develop high-resolution data for national forests. State agencies in California,
Colorado, Florida, Georgia, Indiana, Kansas, Kentucky, Maine, Minnesota, Missouri, Nebraska, New Jersey, North Carolina, Ohio, Texas, Utah, Vermont, and West Virginia are partnering with the USGS to develop high-resolution data for parts of all of their States. Much of the USGS and USFS work is done under contract with the private sector, adding options for other organizations to develop and maintain the NHD.

\section{Partnership Opportunities}

Organizations interested in partnering with the USGS to develop high-resolution data for the NHD sould contact the USGS mapping liaison in their State. The list of liaisons is available at mapping.usgs.gov/www.partnrs/ crreps.html.

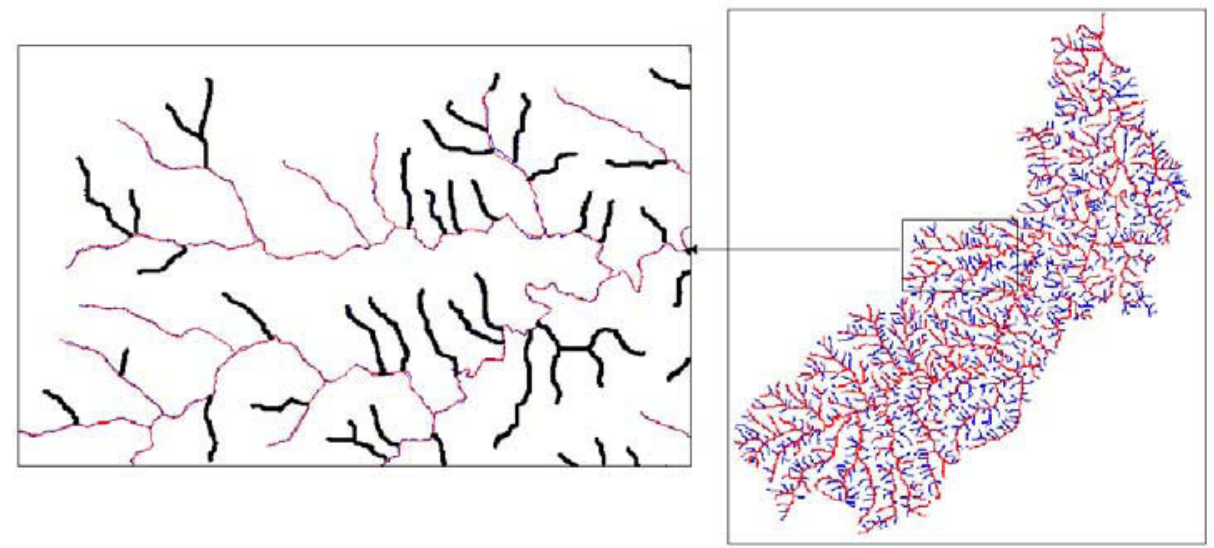

Comparison of medium-resolution NHD data (thin lines) and content added in high-resolution NHD data (thick lines). Big Sandy, Kentucky, subbasin. 